\title{
Classical solutions of sigma models in curved backgrounds by the Poisson-Lie T-plurality
}

\author{
Ladislav Hlavatý, Jan Hýbl, Miroslav Turek
}

April 22, 2018

\begin{abstract}
Faculty of Nuclear Sciences and Physical Engineering, Czech Technical University in Prague, Břehová 7, 11519 Prague 1, Czech Republic

e-mail: hlavaty@fjfi.cvut.cz, tolavy.vitr@centrum.cz, turekm@km1.fjfi.cvut.cz
\end{abstract}

\begin{abstract}
Classical equations of motion for three-dimensional $\sigma$-models in curved background are solved by a transformation that follows from the Poisson-Lie T-plurality and transform them into the equations in the flat background. Transformations of coordinates that make the metric constant are found and used for solving the flat model. The Poisson-Lie transformation is explicitly performed by solving the PDE's for auxiliary functions and finding the relevant transformation of coordinates in the Drinfel'd double. String conditions for the solutions are preserved by the Poisson-Lie transformations. Therefore we are able to specify the type of $\sigma$-model solutions that solve also equations of motion of three dimensional relativistic strings in the curved backgrounds. Simple examples are given
\end{abstract}

\section{Introduction}

Recently a classical solution of equations of motion of a $\sigma$-model in curved background was solved by the T-duality transformation [1]. Poisson-Lie Tplurality transformations provide us with a prescription how to relate a much wider class of $\sigma$-models with apparently different target space geometries. In this paper we are going to present two cases of three-dimensional $\sigma$-models 
that can be solved by this way. Additional conditions on the solutions then produce classical strings in the curved backgrounds.

Klimčík and Ševera in their seminal work [2] described the conditions and procedure for transforming solutions of a $\sigma$-model to those of a dual one. This procedure can be extended in such a way that it enables transformation of the solution of original model to solution of every $\sigma$-model that can be derived from equations on the Drinfel'd double defined by the original $\sigma$-model.

Let us assume that a covariant second order tensor field $F$ is given on a Lie group $G$. The classical action of the $\sigma$-model then is

$$
S_{F}[\phi]=\int d^{2} x \partial_{-} \phi^{\mu} F_{\mu \nu}(\phi) \partial_{+} \phi^{\nu}
$$

where the functions $\phi^{\mu}: \mathbf{R}^{2} \rightarrow \mathbf{R}, \mu=1,2, \ldots, \operatorname{dim} G$ are obtained by the composition $\phi^{\mu}=y^{\mu} \circ \phi$ of a map $\phi: \mathbf{R}^{2} \rightarrow G$ and a coordinate map $y: U_{g} \rightarrow \mathbf{R}^{n}, n=\operatorname{dim} G$ of a neighborhood of an element $\phi\left(x_{+}, x_{-}\right)=g \in G$. The equations of motion have the form

$$
\partial_{-} \partial_{+} \phi^{\mu}+\Gamma_{\nu \lambda}^{\mu} \partial_{-} \phi^{\nu} \partial_{+} \phi^{\lambda}=0
$$

where

$$
\Gamma_{\nu \lambda}^{\mu}:=\frac{1}{2} G^{\mu \rho}\left(F_{\rho \lambda, \nu}+F_{\nu \rho, \lambda}-F_{\nu \lambda, \rho}\right)
$$

and

$$
G_{\mu \nu}=\frac{1}{2}\left(F_{\mu \nu}+F_{\nu \mu}\right), \quad G_{\mu \nu} G^{\nu \rho}=G^{\rho \nu} G_{\nu \mu}=\delta_{\nu}^{\rho}
$$

If $F$ satisfies

$$
\mathcal{L}_{v_{i}}(F)_{\mu \nu}=F_{\mu \kappa} v_{j}^{\kappa} \widetilde{f}_{i}^{j k} v_{k}^{\lambda} F_{\lambda \nu}, i, \mu, \nu=1, \ldots, \operatorname{dim} G
$$

where $v_{i}$ form a basis of left-invariant fields on $G$ and $\tilde{f}_{i}^{j k}$ are structure coefficients of a Lie group $\widetilde{G}, \operatorname{dim} \widetilde{G}=\operatorname{dim} G$, then one can rewrite the equations of motion of the $\sigma$-model as equations for a map to a Drinfel'd double - connected Lie group whose Lie algebra $\mathcal{D}$ admits a decomposition into two subalgebras that are maximally isotropic with respect to a bilinear, symmetric, nondegenerate, ad-invariant form on $\mathcal{D}$. This decomposition $\mathcal{D}=(\mathcal{G} \mid \widetilde{\mathcal{G}})$ is called the Manin triple.

The fact that several decompositions of its Lie algebra $\mathcal{D}$ into Manin triples $(\mathcal{G} \mid \widetilde{\mathcal{G}})$ may exist for one Drinfel'd double leads to the notion of PoissonLie T-plurality [3]. Solutions of the equations of motion for $S_{F}$ then can be 
transformed to solutions of another $\sigma$-model for $S_{\widehat{F}}$ where $\widehat{F}_{\mu \nu}$ is a second order tensor field on a group $\widehat{G}$ that belongs to another decomposition of the same Drinfel'd double.

In this paper we are going to exploit plural decompositions of two sixdimensional Drinfel'd doubles denoted in the classification [4] as DD11 and DD12. The reason for this choice is that the Poisson-Lie transformation relates $\sigma$-models with flat and curved background and meanwhile the equations necessary for performing the transformation are explicitly solvable.

\section{Elements of Poisson-Lie transformation}

The tensors $F$ of dualizable $\sigma$-models, i.e. such that satisfy (4), can be written in terms of right-invariant fields on a Lie group $G$ as

$$
F_{\mu \nu}(y)=e_{\mu}^{a}(g(y)) E_{a b}(g(y)) e_{\nu}^{b}(g(y))
$$

where $y^{i}$ are local coordinates of $g \in G, e_{\mu}^{a}$ are components of right-invariant forms (vielbeins) $e_{\mu}^{a}(g)=\left((\mathrm{d} g) \cdot g^{-1}\right)_{\mu}^{a}$,

$$
E(g)=\left(E_{0}^{-1}+\Pi(g)\right)^{-1}, \quad \Pi(g)=b(g) a(g)^{-1}=-\Pi(g)^{t},
$$

and $a(g), b(g)$ are submatrices of the adjoint representation of the group $G$ on the Lie algebra of the Drinfel'd double ${ }^{1}$

$$
A d(g)^{t}=\left(\begin{array}{cc}
a(g) & 0 \\
b(g) & d(g)
\end{array}\right) .
$$

The Poisson-Lie transformation of the tensor $F$ is defined as follows 3 . Let $\left\{T_{j}, \tilde{T}^{k}\right\}, j, k \in\{1, \ldots, n\}$ be generators of Lie subalgebras $\mathcal{G}, \widetilde{\mathcal{G}}$ of the Manin triple associated with the Lagrangian (11) and $\left\{U_{j}, \tilde{U}^{k}\right\}$ are generators of some other Manin triple $\left(\mathcal{G}_{U} \mid \widetilde{\mathcal{G}}_{U}\right)$ of the same Drinfel'd double related by the $2 n \times 2 n$ transformation matrix as

$$
\left(\begin{array}{c}
\vec{T} \\
\overrightarrow{\tilde{T}}
\end{array}\right)=\left(\begin{array}{ll}
P & T \\
R & S
\end{array}\right)\left(\begin{array}{c}
\vec{U} \\
\overrightarrow{\tilde{U}}
\end{array}\right),
$$

where

$$
\vec{T}=\left(T_{1}, \ldots, T_{n}\right)^{t}, \ldots, \overrightarrow{\tilde{U}}=\left(\tilde{U}^{1}, \ldots, \tilde{U}^{n}\right)^{t}
$$

\footnotetext{
${ }^{1} \mathrm{t}$ denotes transposition.
} 
The transformed model is then given by the Lagrangian of the form (11) but with $E(g)$ replaced by

$$
\widehat{E}_{U}\left(g_{u}\right)=M\left(N+\Pi_{U}\left(g_{u}\right) M\right)^{-1}=\left(\widehat{E}_{0}^{-1}+\Pi_{U}\left(g_{u}\right)\right)^{-1},
$$

where

$$
\widehat{E}_{0}=M N^{-1}, \quad M=S^{t} E_{0}-T^{t}, \quad N=P^{t}-R^{t} E_{0},
$$

and $\Pi_{U}$ is calculated by (6) from the adjoint representation of the group $G_{U}$ generated by $\left\{U_{j}\right\}$.

The relation between the solutions $\phi\left(x_{+}, x_{-}\right)$of the equations of motion of the model given by $F$ and $\widehat{\phi}\left(x_{+}, x_{-}\right)$of the model given by $\widehat{F}$ follows from two possible decompositions of elements $d$ of Drinfel'd double

$$
d=g \cdot \tilde{h}=\widehat{g} \cdot h^{\prime}
$$

where $g \in G, \widetilde{h} \in \widetilde{G}, \widehat{g} \in \widehat{G}, h^{\prime} \in \widetilde{\widehat{G}}$.

The map $\widetilde{h}: \mathbf{R}^{2} \rightarrow \widetilde{G}$ that we need for this transformation satisfies the equations [5]

$$
\begin{aligned}
\left(\left(\partial_{+} \widetilde{h}\right) \cdot \widetilde{h}^{-1}\right)_{j} & =-A_{+, j}:=-v_{j}^{\lambda} F_{\lambda \nu}(\phi) \partial_{+} \phi^{\nu} \\
\left(\left(\partial_{-} \widetilde{h}\right) \cdot \widetilde{h}^{-1}\right)_{j} & =-A_{-, j}:=\partial_{-} \phi^{\lambda} F_{\lambda \nu}(\phi) v_{j}^{\nu}
\end{aligned}
$$

The equations (813) define the Poisson-Lie transformation but their solution is usually very complicated to use them for finding the solutions. Essentially, there are three steps in performing the transformation:

1. You must know a solution $\phi\left(x_{+}, x_{-}\right)$of the $\sigma$-model given by $F$.

2. For the given $\phi\left(x_{+}, x_{-}\right)$you must find $\widetilde{h}\left(x_{+}, x_{-}\right)$i.e. solve the system of PDE's (1213).

3. For given $d\left(x_{+}, x_{-}\right)=\phi\left(x_{+}, x_{-}\right) \cdot \widetilde{h}\left(x_{+}, x_{-}\right) \in D$ you must find the decomposition $d\left(x_{+}, x_{-}\right)=\widehat{\phi}\left(x_{+}, x_{-}\right) \cdot h^{\prime}\left(x_{+}, x_{-}\right)$where $\widehat{\phi}\left(x_{+}, x_{-}\right) \in \widehat{G}$, $h^{\prime}\left(x_{+}, x_{-}\right) \in \widetilde{\widehat{G}}$.

In the next section we shall present two examples of three-dimensional $\sigma-$ models with nontrivial (i.e. curved) backgrounds found in [6] for which all the three steps can be done and the $\sigma$-models can be explicitly solved by the Poisson-Lie transformation. The models are obtained from the Manin triples $(5 \mid 1) \cong\left(6_{0} \mid 1\right)$ and $(4 \mid 1) \cong\left(6_{0} \mid 2\right)$ (the notation will be explained below) that are decompositions of the Drinfel'd doubles DD11 and DD12 respectively, and relate $\sigma$-models in flat and curved backgrounds. 


\section{Solution of the $\sigma$-models corresponding to DD11}

The first two $\sigma$-models we are going to investigate are given by the tensors

$$
F_{\mu \nu}(y)=\left(\begin{array}{lll}
0 & 0 & \kappa e^{-y_{1}} \\
0 & \kappa e^{-2 y_{1}} & 0 \\
\kappa e^{-y_{1}} & 0 & 0
\end{array}\right), \quad \kappa \in R,
$$

and

$$
\widehat{F}_{\mu \nu}(\widehat{y})=\left(\begin{array}{lll}
\frac{1}{\kappa} e^{-2 \widehat{y_{3}}} & \frac{1}{\kappa} e^{-2 \widehat{y_{3}}} & \frac{\kappa}{2} e^{\widehat{y_{3}}} \\
\frac{1}{\kappa} e^{-2 \widehat{y_{3}}} & \frac{1}{\kappa} e^{-2 \widehat{y_{3}}} & -\frac{\kappa}{2} e^{\widehat{y_{3}}} \\
\frac{\kappa}{2} e^{\widehat{y_{3}}} & -\frac{\kappa}{2} e^{\widehat{y_{3}}} & 0
\end{array}\right)
$$

Both these tensors are symmetric so that the models are torsionless and the tensors $F$ and $\widehat{F}$ can be interpreted as metrics. The first metric is flat. The Ricci tensor of the second metric is nontrivial so that the background of the $\sigma$-model given by (15) is curved but its Gauss curvature is zero.

The tensor $F$ can be obtained from the formulas (5) (7) using

$$
E_{0}=\left(\begin{array}{ccc}
0 & 0 & \kappa \\
0 & \kappa & 0 \\
\kappa & 0 & 0
\end{array}\right)=\left.F\right|_{y=0}, \quad \kappa \in R,
$$

and Manin triple $(5 \mid 1)$ that is a decomposition $\mathcal{D}=(\mathcal{G} \mid \widetilde{\mathcal{G}})$ of the Drinfel'd double DD11 where $\mathcal{G}=$ Bianchi 5 given by the commutation relations

$$
\left[T_{1}, T_{2}\right]=-T_{2}, \quad\left[T_{2}, T_{3}\right]=0, \quad\left[T_{3}, T_{1}\right]=T_{3},
$$

and $\widetilde{\mathcal{G}}$ abelian.

The tensor $\widehat{F}$ is obtained from $\widehat{E_{0}}=\left.\widehat{F}\right|_{\widehat{y}=0}$ and another decomposition of DD11, namely $\left(6_{0} \mid 1\right)$ where $\mathcal{G}=$ Bianchi $6_{0}$ given by the commutation relations

$$
\left[U_{1}, U_{2}\right]=0, \quad\left[U_{2}, U_{3}\right]=U_{1}, \quad\left[U_{3}, U_{1}\right]=-U_{2},
$$

and $\widetilde{\mathcal{G}}$ abelian. 
Relation between these two decompositions is given by (8) where

$$
\left(\begin{array}{ll}
P & T \\
R & S
\end{array}\right)=\left(\begin{array}{llllll}
0 & 0 & -1 & 0 & 0 & 0 \\
0 & 0 & 0 & 1 & 1 & 0 \\
-1 & 1 & 0 & 0 & 0 & 0 \\
0 & 0 & 0 & 0 & 0 & -1 \\
\frac{1}{2} & \frac{1}{2} & 0 & 0 & 0 & 0 \\
0 & 0 & 0 & -\frac{1}{2} & \frac{1}{2} & 0
\end{array}\right)
$$

Inserting (16) and (19) into (10) we get $\widehat{E_{0}}=\left.\widehat{F}\right|_{\widehat{y}=0}$ and when the corresponding matrix (9) is inserted into (5) we get the tensor $\widehat{F}(\widehat{y})$. It means that the equations of motion for the $\sigma$-models given by both (14) and (15) can be rewritten as the same equation on the six-dimensional Drinfel'd double DD11. In other words, the $\sigma$-models are Poisson-Lie T-plural. In the following subsections we shall present the Poisson-Lie transformation between solutions of their equations of motion.

\subsection{Solution of the flat model}

Even though we know that the model given by the tensor (14) lives in the flat background it is not straightforward to solve the equation of motion because the Christoffel symbols are not zero. To find the functions $\phi^{\mu}\left(x_{+}, x_{-}\right)$that solve the equation of motion we must express $\phi^{\mu}$ in terms of coordinates for which the metric become constant. Transformation of coordinates [7]

$$
\begin{gathered}
\xi_{1}=-e^{-\phi^{1}} \\
\xi_{2}=\phi^{2} e^{-\phi^{1}} \\
\xi_{3}=\phi^{3}+\frac{1}{2}\left(\phi^{2}\right)^{2} e^{-\phi^{1}}
\end{gathered}
$$

brings the metric (14) to constant form $G^{\prime}(\xi)=E_{0}$ and equations of motion transform to the wave equations so that

$$
\xi_{j}\left(x_{+}, x_{-}\right)=W_{j}\left(x_{+}\right)+Y_{j}\left(x_{-}\right)
$$


with arbitrary $W_{j}$ and $Y_{j}$. Functions $\phi^{\mu}\left(x_{+}, x_{-}\right)$that solve the equations of motion for $S_{F}[\phi]$ then follow from (20) and (21).

$$
\begin{aligned}
& \phi^{1}\left(x_{+}, x_{-}\right)=-\ln \left(-W_{1}-Y_{1}\right), \\
& \phi^{2}\left(x_{+}, x_{-}\right)=-\frac{W_{2}+Y_{2}}{W_{1}+Y_{1}}, \\
& \phi^{3}\left(x_{+}, x_{-}\right)=W_{3}+Y_{3}+\frac{\left(W_{2}+Y_{2}\right)^{2}}{2\left(W_{1}+Y_{1}\right)} .
\end{aligned}
$$

where $W_{j}=W_{j}\left(x_{+}\right), Y_{j}=Y_{j}\left(x_{-}\right)$.

This finishes the first step, namely obtaining the solution of the flat $\sigma-$ model. The second step of the Poisson-Lie transformation requires solving the system of PDEs (1213) for auxiliary functions $\widetilde{h}$.

\subsection{Solution of the system $(12,13)$}

The coordinates $\widetilde{h}_{\nu}$ in the Abelian group $\widetilde{G}$ can be chosen so that the lefthand sides of the equations (1213) are just $\partial_{ \pm} \widetilde{h}_{\nu}$. The right-hand sides are

$$
\begin{gathered}
A_{+}=\left(\begin{array}{c}
\kappa \phi^{3} e^{-\phi^{1}} \partial_{+} \phi^{1}+\kappa \phi^{2} e^{-2 \phi^{1}} \partial_{+} \phi^{2}+\kappa e^{-\phi^{1}} \partial_{+} \phi^{3} \\
\kappa e^{-2 \phi^{1}} \partial_{+} \phi^{2} \\
\kappa e^{-\phi^{1}} \partial_{+} \phi^{1}
\end{array}\right) \\
A_{-}=\left(\begin{array}{c}
-\kappa \phi^{3} e^{-\phi^{1}} \partial_{-} \phi^{1}-\kappa \phi^{2} e^{-2 \phi^{1}} \partial_{-} \phi^{2}-\kappa e^{-\phi^{1}} \partial_{-} \phi^{3} \\
-\kappa e^{-2 \phi^{1}} \partial_{-} \phi^{2} \\
-\kappa e^{-\phi^{1}} \partial_{-} \phi^{1}
\end{array}\right)
\end{gathered}
$$

and they become rather involved expressions in $W\left(x_{+}\right)$and $Y\left(x_{-}\right)$for the solution $\phi^{\mu}\left(x_{+}, x_{-}\right)$found in the previous subsection. Nevertheless, the equa- 
tions (1213) can be solved and the general solution is

$$
\begin{aligned}
& \tilde{h}_{1}\left(x_{+}, x_{-}\right)=\kappa\left[Y_{1}\left(x_{-}\right) W_{3}\left(x_{+}\right)-Y_{3}\left(x_{-}\right) W_{1}\left(x_{+}\right)+\gamma\left(x_{+}\right)+\delta\left(x_{-}\right)\right] \\
& \tilde{h}_{2}\left(x_{+}, x_{-}\right)=\kappa\left[Y_{1}\left(x_{-}\right) W_{2}\left(x_{+}\right)-Y_{2}\left(x_{-}\right) W_{1}\left(x_{+}\right)+\alpha\left(x_{+}\right)+\beta\left(x_{-}\right)\right] \\
& \tilde{h}_{3}\left(x_{+}, x_{-}\right)=\kappa\left[Y_{1}\left(x_{-}\right)-W_{1}\left(x_{+}\right)\right]
\end{aligned}
$$

where

$$
\begin{aligned}
\alpha^{\prime} & =W_{1} W_{2}^{\prime}-W_{2} W_{1}^{\prime} \\
\beta^{\prime} & =Y_{2} Y_{1}^{\prime}-Y_{1} Y_{2}^{\prime}
\end{aligned}
$$

(primes denote differentiation) and

$$
\begin{aligned}
\gamma^{\prime} & =W_{1} W_{3}^{\prime}-W_{3} W_{1}^{\prime} \\
\delta^{\prime} & =Y_{3} Y_{1}^{\prime}-Y_{1} Y_{3}^{\prime}
\end{aligned}
$$

\subsection{Plural decompositions of elements of the Drinfel'd double}

The final step in the Poisson-Lie transformation follows from the possibility of rewriting an element of the Drinfel'd double

$$
d\left(x_{+}, x_{-}\right)=\phi\left(x_{+}, x_{-}\right) \cdot \widetilde{h}\left(x_{+}, x_{-}\right)
$$

where $\phi\left(x_{+}, x_{-}\right) \in G, \widetilde{h}\left(x_{+}, x_{-}\right) \in \widetilde{G}$, to the form

$$
d\left(x_{+}, x_{-}\right)=\widehat{\phi}\left(x_{+}, x_{-}\right) \cdot h^{\prime}\left(x_{+}, x_{-}\right), \quad \widehat{\phi}\left(x_{+}, x_{-}\right) \in \widehat{G}, h^{\prime}\left(x_{+}, x_{-}\right) \in \widetilde{\widehat{G}} .
$$

As all the goups are solvable we can write all group elements as product of elements of one-parametric subgroups

$$
\mathrm{e}^{\phi^{1} T_{1}} \mathrm{e}^{\phi^{2} T_{2}} \mathrm{e}^{\phi^{3} T_{3}} \mathrm{e}^{\widetilde{h}_{1} \widetilde{T}^{1}} \mathrm{e}^{\widetilde{h}_{2} \widetilde{T}^{2}} \mathrm{e}^{\widetilde{h}_{3} \widetilde{T}^{3}}=\mathrm{e}^{\widehat{\phi}^{3} U_{3}} \mathrm{e}^{\widehat{\phi}^{2} U_{2}} \mathrm{e}^{\widehat{\phi}^{1} U_{1}} \mathrm{e}^{h_{3}^{\prime} \widetilde{U}^{3}} \mathrm{e}^{h_{2}^{\prime} \widetilde{U}^{2}} \mathrm{e}^{h_{1}^{\prime} \widetilde{U}^{1}}
$$

where $T_{j}, \widetilde{T}^{k}$ are elements of basis $(5 \mid 1)$ and $U_{j}, \widetilde{U}^{k}$ are elements of basis $\left(6_{0} \mid 1\right)$. Note the reverse order of subgroups on the right-hand side. Inverting the matrix (19) we get from (8) the right-hand side in the form

$$
e^{\widehat{\phi}^{3}\left(-T_{1}\right)} e^{\widehat{\phi}^{2}\left(\frac{1}{2} T_{3}+\widetilde{T}^{2}\right)} e^{\widehat{\phi}^{1}\left(-\frac{1}{2} T_{3}+\widetilde{T}^{2}\right)} e^{h_{3}^{\prime}\left(-\widetilde{T}^{1}\right)} e^{h_{2}^{\prime}\left(\frac{1}{2} T_{2}+\widetilde{T}^{3}\right)} e^{h_{1}^{\prime}\left(\frac{1}{2} T_{2}-\widetilde{T}^{3}\right)}
$$


and the two possible decompositions of the Drinfel'd double elements yield an equation for $\widehat{\phi}_{\mu}$ and $h^{\prime \nu}$ in terms of $\widetilde{h}_{\lambda}$ and $\phi^{\rho}$. To solve it might be rather complicated in general but in this case when the only nonzero Lie products of $(5 \mid 1)$ are

$$
\begin{aligned}
& {\left[T_{1}, T_{2}\right]=-T_{2}, \quad\left[T_{1}, T_{3}\right]=-T_{3}, \quad\left[T_{1}, \widetilde{T}^{2}\right]=\widetilde{T}^{2},} \\
& {\left[T_{1}, \widetilde{T}^{3}\right]=\widetilde{T}^{3}, \quad\left[T_{2}, \widetilde{T}^{2}\right]=-\widetilde{T}^{1}, \quad\left[T_{3}, \widetilde{T}^{3}\right]=-\widetilde{T}^{1},}
\end{aligned}
$$

it can be done. When we have used the reverse order of subgroups on the lefthand side of (28) then we can use the simple form of the Baker-CampbellHausdorff formula

$$
\mathrm{e}^{A} \mathrm{e}^{B}=\mathrm{e}^{B} \mathrm{e}^{A} \mathrm{e}^{[A, B]}
$$

since in relevant cases $[[A, B], A]=0=[[A, B], B]$. By repeated application of this formula we get

$$
\begin{aligned}
\widehat{\phi}^{1} & =-\phi^{3}+\frac{1}{2} \tilde{h}_{2} \\
\widehat{\phi}^{2} & =\phi^{3}+\frac{1}{2} \tilde{h}_{2} \\
\widehat{\phi}^{3} & =-\phi^{1} \\
h_{1}^{\prime} & =-\frac{1}{2} \tilde{h}_{3}+\phi^{2} \\
h_{2}^{\prime} & =\frac{1}{2} \tilde{h}_{3}+\phi^{2} \\
h_{3}^{\prime} & =-\tilde{h}_{1}+\tilde{h}_{2} \phi^{2}
\end{aligned}
$$

Inserting (22) and (25) into (30) we get the solution of the equations of motion for the $\sigma$-model in the curved background (15)

$$
\begin{aligned}
\widehat{\phi}^{1}\left(x_{+}, x_{-}\right)= & \frac{1}{2} \kappa\left(Y_{1}\left(x_{-}\right) W_{2}\left(x_{+}\right)-Y_{2}\left(x_{-}\right) W_{1}\left(x_{+}\right)\right)-\left(W_{3}\left(x_{+}\right)+Y_{3}\left(x_{-}\right)\right) \\
& -\frac{1}{2} \frac{\left(W_{2}\left(x_{+}\right)+Y_{2}\left(x_{+}\right)\right)^{2}}{\left(W_{1}\left(x_{+}\right)+Y_{1}\left(x_{-}\right)\right)}+\frac{1}{2} \kappa\left(\alpha\left(x_{+}\right)+\beta\left(x_{-}\right)\right), \\
\widehat{\phi}^{2}\left(x_{+}, x_{-}\right)= & \frac{1}{2} \kappa\left(Y_{1}\left(x_{-}\right) W_{2}\left(x_{+}\right)-Y_{2}\left(x_{-}\right) W_{1}\left(x_{+}\right)\right)+\left(W_{3}\left(x_{+}\right)+Y_{3}\left(x_{-}\right)\right) \\
& +\frac{1}{2} \frac{\left(W_{2}\left(x_{+}\right)+Y_{2}\left(x_{-}\right)\right)^{2}}{W 1\left(x_{+}\right)+Y_{1}\left(x_{-}\right)}+\frac{1}{2} \kappa\left(\alpha\left(x_{+}\right)+\beta\left(x_{-}\right)\right), \\
\widehat{\phi}^{3}\left(x_{+}, x_{-}\right)= & \ln \left(-W_{1}\left(x_{+}\right)-Y_{1}\left(x_{-}\right)\right) .
\end{aligned}
$$


where $\alpha, \beta$ satisfy (26) $)$. An example of a simple solution is

$$
\begin{aligned}
& \widehat{\phi}^{1}\left(x_{+}, x_{-}\right)=-\frac{1}{2}\left(W_{1}\left(x_{+}\right)+Y_{1}\left(x_{-}\right)\right) \\
& \widehat{\phi}^{2}\left(x_{+}, x_{-}\right)=\frac{1}{2}\left(W_{1}\left(x_{+}\right)+Y_{1}\left(x_{-}\right)\right) \\
& \widehat{\phi}^{3}\left(x_{+}, x_{-}\right)=\ln \left(-W_{1}\left(x_{+}\right)-Y_{1}\left(x_{-}\right)\right)
\end{aligned}
$$

obtained from (32) for $W_{2}=-W_{1}, Y_{2}=-Y_{1}, W_{3}=0, Y_{3}=0, W_{1}$ a $Y_{1}$ arbitrary.

\section{Solution of the $\sigma$-models corresponding to DD12}

The $\sigma$-models corresponding to the decompositions (4|1) and $\left(6_{0} \mid 2\right)$ of the Drinfel'd double DD12 are given by the tensors

$$
F_{\mu \nu}(y)=\left(\begin{array}{lll}
0 & \kappa y_{1} e^{-y_{1}} & \kappa e^{-y_{1}} \\
\kappa y_{1} e^{-y_{1}} & \kappa e^{-2 y_{1}} & 0 \\
\kappa e^{-y_{1}} & 0 & 0
\end{array}\right), \quad \kappa \in R
$$

and

$$
\widehat{F}_{\mu \nu}(\widehat{y})=\left(\begin{array}{lll}
\frac{1}{\kappa} e^{2 \widehat{y} 3} & -\frac{1}{\kappa} e^{2 \widehat{y_{3}}} & \frac{1}{2} \kappa e^{-\widehat{y}_{3}}+e^{\widehat{y}_{3}} \widehat{y}_{3} \\
-\frac{1}{\kappa} e^{2 \widehat{y}_{3}} & \frac{1}{\kappa} e^{2 \widehat{y}_{3}} & \frac{1}{2} \kappa e^{-\widehat{y}_{3}}-e^{\widehat{y}_{3}} \widehat{y}_{3} \\
\frac{1}{2} \kappa e^{-\widehat{y}_{3}}-e^{\widehat{y_{3}} \widehat{y}_{3}} & \frac{1}{2} \kappa e^{-\widehat{y}_{3}}+e^{\widehat{y}_{3}} \widehat{y}_{3} & -\kappa \widehat{y}_{3}^{2}
\end{array}\right)
$$

Both these tensors are torsionless and their symmetric parts can be interpreted as metrics. The first metric is flat. The Ricci tensor of the second metric is nontrivial but the Gauss curvature is zero.

The tensor $F$ can be obtained from the formulas (5-7) using

$$
E_{0}=\left(\begin{array}{ccc}
0 & 0 & \kappa \\
0 & \kappa & 0 \\
\kappa & 0 & 0
\end{array}\right)
$$


and the Manin triple $(4 \mid 1)$, i.e. $\mathcal{G}=$ Bianchi 4 given by the commutation relations

$$
\left[T_{1}, T_{2}\right]=T_{3}-T_{2}, \quad\left[T_{2}, T_{3}\right]=0, \quad\left[T_{3}, T_{1}\right]=T_{3},
$$

and $\widetilde{\mathcal{G}}$ abelian.

The tensor $\widehat{F}$ is obtained from $\widehat{E_{0}}=\left.\widehat{F}\right|_{\widehat{y}=0}$ and the Manin triple $\left(6_{0} \mid 2\right)$, where $\mathcal{G}=$ Bianchi $6_{0}$ is given by the commutation relations

$$
\left[U_{1}, U_{2}\right]=0, \quad\left[U_{2}, U_{3}\right]=U_{1}, \quad\left[U_{3}, U_{1}\right]=-U_{2},
$$

and $\widetilde{\mathcal{G}}=$ Bianchi2 given by the commutation relations

$$
\left[\widetilde{U}^{1}, \widetilde{U}^{2}\right]=\widetilde{U}^{3}, \quad\left[\widetilde{U}^{2}, \widetilde{U}^{3}\right]=0, \quad\left[\widetilde{U}^{3}, \widetilde{U}^{1}\right]=0 .
$$

Relation between these two decompositions is given by (8) where

$$
\left(\begin{array}{ll}
P & T \\
R & S
\end{array}\right)=\left(\begin{array}{llllll}
0 & 0 & 1 & 0 & 0 & 0 \\
0 & 0 & 0 & 1 & -1 & 0 \\
1 & 1 & 0 & 0 & 0 & 0 \\
0 & 0 & 0 & 0 & 0 & 1 \\
\frac{1}{2} & -\frac{1}{2} & 0 & 0 & 0 & 0 \\
0 & 0 & 0 & \frac{1}{2} & \frac{1}{2} & 0
\end{array}\right)
$$

Inserting (136) and (401) into (101) we get $\widehat{E}_{0}=\left.\widehat{F}\right|_{\widehat{y}=0}$ and when the matrix $E_{U}\left(g_{u}\right)$ is inserted into (5) the tensor $\widehat{F}$ is obtained. It means that the equations of motion for the $\sigma-$ models given by both (34) and (35) can be rewritten as the same equation on the Drinfel'd double DD12. In the following subsections we shall present the Poisson-Lie transformation between $\sigma$-models given by $F$ and $\widehat{F}$.

\subsection{Solution of the flat model}

To find the functions $\phi^{\mu}\left(x_{+}, x_{-}\right)$that solve the equation of motion of the flat $\sigma$-model we must express $\phi^{\mu}$ in terms of coordinates $\xi$

$$
\begin{gathered}
\xi_{1}=-e^{-\phi^{1}} \\
\xi_{2}=e^{-\phi^{1}}\left(1+\phi^{2}\right)+\phi^{1}-1 \\
\xi_{3}=\frac{1}{2} e^{-\phi^{1}}\left(1+\phi^{2}\right)^{2}+\phi^{3}+\phi^{1} \phi^{2}-\phi^{2}+\phi^{1}-\frac{1}{2} e^{\phi^{1}}
\end{gathered}
$$


for which the metric become constant and equations of motion transform to the wave equations. Solution of the equations of motion for $S_{F}[\phi]$ that follow from (41) and (21) then is

$$
\begin{aligned}
& \phi^{1}\left(x_{+}, x_{-}\right)=-\ln \left(-W_{1}-Y_{1}\right), \\
& \phi^{2}\left(x_{+}, x_{-}\right)=-1-\frac{1+W_{2}+Y_{2}+\ln \left(-W_{1}-Y_{1}\right)}{W_{1}+Y_{1}}, \\
& \phi^{3}\left(x_{+}, x_{-}\right)=-1+W_{3}+Y_{3}+\frac{1}{2\left(W_{1}+Y_{1}\right)}\left[-1+\left(W_{2}+Y_{2}\right)^{2}-\left(1+\ln \left(-W_{1}-Y_{1}\right)\right)^{2}\right] .
\end{aligned}
$$

where $W_{j}=W_{j}\left(x_{+}\right), Y_{j}=Y_{j}\left(x_{-}\right)$are arbitrary functions.

As the next step we have to solve the equations for the auxiliary functions $\widetilde{h}_{\nu}\left(x_{+}, x_{-}\right)$.

\subsection{Solution of the system (12,13)}

The coordinates $\widetilde{h}_{\nu}$ in the Abelian group $\widetilde{G}$ can be chosen so that the lefthand sides of the equations (1213) are just $\partial_{ \pm} \widetilde{h}_{\nu}$. The right-hand sides in this case are

$$
A_{+}=\left(\begin{array}{c}
\kappa e^{-\phi^{1}}\left(\left(\phi^{2} \phi^{1}-\phi^{2}+\phi^{3}\right) \partial_{+} \phi^{1}+\left(\phi^{1}+e^{-\phi^{1}} \phi^{2}\right) \partial_{+} \phi^{2}+\partial_{+} \phi^{3}\right) \\
\kappa e^{-\phi^{1}}\left(\phi^{1} \partial_{+} \phi^{1}+e^{-\phi^{1}} \partial_{+} \phi^{2}\right) \\
\kappa e^{-\phi^{1}} \partial_{+} \phi^{1}
\end{array}\right)
$$

$$
A_{-}=\left(\begin{array}{c}
-\kappa e^{-\phi^{1}}\left(\left(\phi^{2} \phi^{1}-\phi^{2}+\phi^{3}\right) \partial_{-} \phi^{1}+\left(\phi^{1}+e^{-\phi^{1}} \phi^{2}\right) \partial_{-} \phi^{2}+\partial_{-} \phi^{3}\right) \\
-\kappa e^{-\phi^{1}}\left(\phi^{1} \partial_{-} \phi^{1}+e^{-\phi^{1}} \partial_{-} \phi^{2}\right) \\
-\kappa e^{-\phi^{1}} \partial_{-} \phi^{1}
\end{array}\right)
$$


where the functions $\phi^{\mu}\left(x_{+}, x_{-}\right)$are given by (42). The general solution of the equations (1213) is

$$
\begin{aligned}
\tilde{h}_{1}= & \kappa\left[Y_{2}\left(x_{-}\right)-W_{2}\left(x_{+}\right)+W_{1}\left(x_{+}\right)\left(Y_{2}\left(x_{-}\right)-Y_{3}\left(x_{-}\right)\right)\right. \\
& \left.-Y_{1}\left(x_{-}\right)\left(W_{2}\left(x_{+}\right)-W_{3}\left(x_{+}\right)\right)-\alpha\left(x_{+}\right)-\beta\left(x_{-}\right)+\gamma\left(x_{+}\right)+\delta\left(x_{-}\right)\right] \\
\tilde{h}_{2}= & \kappa\left[W_{2}\left(x_{+}\right) Y_{1}\left(x_{-}\right)-W_{1}\left(x_{+}\right) Y_{2}\left(x_{-}\right)+\alpha\left(x_{+}\right)+\beta\left(x_{-}\right)\right] \\
\tilde{h}_{3}= & \kappa\left[Y_{1}\left(x_{-}\right)-W_{1}\left(x_{+}\right)\right]
\end{aligned}
$$

where the functions $\alpha, \beta, \gamma, \delta$ satisfy (26), (27).

\subsection{Plural decomposition of elements of the Drinfel'd double}

The possibility of writing an element of the Drinfel'd double in the decompositions $(4 \mid 1)$ and $\left(6_{0} \mid 2\right)$

$$
\mathrm{e}^{\phi^{1} T_{1}} \mathrm{e}^{\phi^{2} T_{2}} \mathrm{e}^{\phi^{3} T_{3}} \mathrm{e}^{\widetilde{h}_{1} \widetilde{T}^{1}} \mathrm{e}^{\widetilde{h}_{2} \widetilde{T}^{2}} \mathrm{e}^{\widetilde{h}_{3} \widetilde{T}^{3}}=\mathrm{e}^{\widehat{\phi}^{3} U_{3}} \mathrm{e}^{\phi^{2} U_{2}} \mathrm{e}^{\widehat{\phi}^{1}} U_{1} \mathrm{e}^{h_{3}^{\prime} \widetilde{U}^{3}} \mathrm{e}^{h_{2}^{\prime} \widetilde{U}^{2}} \mathrm{e}^{h_{1}^{\prime} \widetilde{U}^{1}} .
$$

yield an equation for $\widehat{\phi}_{\mu}$ and $h^{\prime \nu}$ in terms of $\widetilde{h}_{\lambda}$ and $\phi^{\rho}$. Inverting the matrix (40) we get from (8) the right-hand side in the form

$$
e^{\widehat{\phi}^{3} T_{1}} e^{\widehat{\phi}^{2}\left(\frac{1}{2} T_{3}-\widetilde{T}^{2}\right)} e^{\widehat{\phi}^{1}\left(\frac{1}{2} T_{3}+\widetilde{T}^{2}\right)} e^{h_{3}^{\prime} \widetilde{T}^{1}} e^{h_{2}^{\prime}\left(-\frac{1}{2} T_{2}+\widetilde{T}^{3}\right)} e^{h_{1}^{\prime}\left(\frac{1}{2} T_{2}+\widetilde{T}^{3}\right)} .
$$

The only nonzero Lie products of (4|1) are

$$
\begin{gathered}
{\left[T_{1}, T_{2}\right]=T_{3}-T_{2}, \quad\left[T_{3}, T_{1}\right]=T_{3},} \\
{\left[T_{1}, \widetilde{T}^{2}\right]=\widetilde{T}^{2}, \quad\left[T_{1}, \widetilde{T}^{3}\right]=-\widetilde{T}^{2}+\widetilde{T}^{3},} \\
{\left[T_{2}, \widetilde{T}^{2}\right]=-\widetilde{T}^{1}, \quad\left[T_{2}, \widetilde{T}^{3}\right]=\widetilde{T}^{1}, \quad\left[T_{3}, \widetilde{T}^{3}\right]=-\widetilde{T}^{1}}
\end{gathered}
$$

and when we have used the reverse order of subgroups on the left-hand side of (28) only the simple form of the Baker-Campbell-Hausdorff formula (29) is necessary in relevant cases. We get

$$
\begin{aligned}
\widehat{\phi}^{1} & =\phi^{3}+\frac{1}{2} \tilde{h}_{2} \\
\widehat{\phi}^{2} & =\phi^{3}-\frac{1}{2} \tilde{h}_{2} \\
\widehat{\phi}^{3} & =\phi^{1}
\end{aligned}
$$




$$
\begin{aligned}
& h_{1}^{\prime}=\frac{1}{2} \tilde{h}_{3}+\phi^{2} \\
& h_{2}^{\prime}=\frac{1}{2} \tilde{h}_{3}-\phi^{2} \\
& h_{3}^{\prime}=\tilde{h}_{1}-\tilde{h}_{2} \phi^{2}+\frac{1}{2} \phi^{2} \phi^{2}+\frac{1}{2} \phi^{2} \tilde{h}_{3}-\frac{1}{8} \tilde{h}_{3} \tilde{h}_{3}
\end{aligned}
$$

Inserting (42) and (45) into (48) we get the solution of the equations of motion for the $\sigma$-model given by the action $S_{\widehat{F}}$ for $\widehat{F}$ given by (35).

$$
\begin{aligned}
\widehat{\phi}^{1}\left(x_{+}, x_{-}\right)= & -1+\left(W_{3}+Y_{3}\right)+\frac{1}{2} \kappa\left[W_{2} Y_{1}-W_{1} Y_{2}+\alpha+\beta\right]+ \\
& \frac{1}{2\left(W_{1}+Y_{1}\right)}\left[-2+\left(W_{2}+Y_{2}\right)^{2}-2 \ln \left(-W_{1}-Y_{1}\right)-\ln ^{2}\left(-W_{1}-Y_{1}\right)\right] \\
\widehat{\phi}^{2}\left(x_{+}, x_{-}\right)= & -1+\left(W_{3}+Y_{3}\right)-\frac{1}{2} \kappa\left[W_{2} Y_{1}-W_{1} Y_{2}+\alpha+\beta\right]+ \\
& \frac{1}{2\left(W_{1}+Y_{1}\right)}\left[-2+\left(W_{2}+Y_{2}\right)^{2}-2 \ln \left(-W_{1}-Y_{1}\right)-\ln ^{2}\left(-W_{1}-Y_{1}\right)\right] \\
\widehat{\phi}^{3}\left(x_{+}, x_{-}\right)= & -\ln \left(-W_{1}-Y_{1}\right)
\end{aligned}
$$

where $W_{j}=W_{j}\left(x_{+}\right), Y_{j}=Y_{j}\left(x_{-}\right), \alpha=\alpha\left(x_{+}\right), \beta=\beta\left(x_{-}\right)$and $\alpha, \beta$ satisfy (26). A simple solution dependent on both $x_{+}$and $x_{-}$is

$$
\begin{aligned}
& \widehat{\phi}^{1}=-\frac{1}{2}\left(W_{2}+Y_{2}\right)^{2}+Y_{2} \\
& \widehat{\phi}^{2}=-\frac{1}{2}\left(W_{2}+Y_{2}\right)^{2}+W_{2} \\
& \widehat{\phi}^{3}=0
\end{aligned}
$$

obtained from (50) for $\kappa=1, W_{1}=0, Y_{1}=-1, W_{3}=\frac{W_{2}}{2}, Y_{3}=\frac{Y_{2}}{2}, W_{2}$ a $Y_{2}$ arbitrary.

\section{String solutions}

It is well known that the string action can be replaced by the $\sigma$-model (Polyakov) action (11) if an additional constraint for the $\sigma$-model solutions

$$
\partial_{a} \phi^{\mu} G_{\mu \nu}(\phi) \partial_{b} \phi^{\nu}=\eta_{a b} e^{\omega}
$$


is required where

$$
\eta=\left(\begin{array}{ll}
0 & 1 \\
1 & 0
\end{array}\right)
$$

and $\omega$ is a function of $x_{+}, x_{-}$. It is easy to see that for the flat models given by (14) or (34) this condition yields

$$
\begin{gathered}
2 W_{1}^{\prime}\left(x_{+}\right) W_{3}^{\prime}\left(x_{+}\right)+W_{2}^{\prime}\left(x_{+}\right) W_{2}^{\prime}\left(x_{+}\right)=0, \\
2 Y_{1}^{\prime}\left(x_{-}\right) Y_{3}^{\prime}\left(x_{-}\right)+Y_{2}^{\prime}\left(x_{-}\right) Y_{2}^{\prime}\left(x_{-}\right)=0 .
\end{gathered}
$$

We have checked that these conditions remain preserved for the curved models as well so that the string solutions both in the flat and curved backgrounds can be obtained by inserting a solution of (153), (54) into (22), (132), (42) and (50).

An example of solution of (53), (54) is

$$
\begin{array}{r}
W_{2}\left(x_{+}\right)=k_{1} W_{1}\left(x_{+}\right)+k_{0}, \quad Y_{2}\left(x_{-}\right)=c_{1} Y_{1}\left(x_{-}\right)+c_{0}, \\
W_{3}\left(x_{+}\right)=-\frac{1}{2} k_{1}^{2} W_{1}\left(x_{+}\right)+k_{0}, \quad Y_{3}\left(x_{-}\right)=-\frac{1}{2} c_{1}^{2} Y_{1}\left(x_{-}\right)+c_{0},
\end{array}
$$

giving

$$
\alpha\left(x_{+}\right)=-k_{0} W_{1}\left(x_{+}\right), \quad \beta\left(x_{-}\right)=c_{0} Y_{1}\left(x_{-}\right)+c_{2},
$$

where $k_{j}, c_{j}$ are constants and $W_{1}, Y_{1}$ arbitrary functions. Other solutions are three-dimensional open strings in the light-cone gauge

$$
\xi_{1}\left(x_{+}, x_{-}\right)=W_{1}\left(x_{+}\right)+Y_{1}\left(x_{-}\right)=2 k p^{+} \tau=\sqrt{2} k p^{+}\left(x_{+}+x_{-}\right)
$$

i.e.

$$
\begin{gathered}
W_{1}(x)=Y_{1}(x)=\sqrt{2} k p^{+} x \\
W_{2}(x)=Y_{2}(x)=\frac{1}{2} X_{2}+\sqrt{k} \alpha_{0} x+i \sqrt{\frac{k}{2}} \sum_{n \neq 0} \frac{1}{n} \alpha_{n} \mathrm{e}^{-i n \sqrt{2} x} \\
W_{3}(x)=Y_{3}(x)=\frac{1}{2} X^{-}-\frac{i}{2 p^{+}} \sum_{n \neq 0} \frac{1}{n} L_{n} \mathrm{e}^{-i n \sqrt{2} x}
\end{gathered}
$$

and

$$
\alpha(x)=-\beta(x)-C=k p^{+}\left(-\frac{X_{2}}{\sqrt{2}} x+\sqrt{k} \sum_{n \neq 0} \alpha_{n}\left(\frac{i x}{n}+\frac{\sqrt{2}}{n^{2}}\right) \mathrm{e}^{-i n \sqrt{2} x}\right),
$$

where

$$
L_{n}=\frac{1}{2} \sum_{p \in \mathbf{Z}} \alpha_{p} \alpha_{n-p}
$$

and $k, p^{+}, X_{2}, X^{-}, \alpha_{n}, C$ are constants. 


\section{Conclusions}

Poisson-Lie transformation between classical solutions of $\sigma$-models can be explicitly performed for the Drinfel'd doubles with sufficiently simple algebraic structure. We have used this fact in solution of equations of motion of three-dimensional $\sigma$-models in the curved backgrounds (15) and (35). These models are by construction dual to flat ones.

We have transformed group coordinates of the dual flat models to those for which their metrics are constant so that solution of the flat $\sigma$-model in the latter coordinates reduces to the solution of the wave equation. Afterwards we had to solve the equations for the auxiliary functions necessary for rewriting the equations for the $\sigma$-model to equations on the Drinfel'd double. Because one of the subgroups of the decompositions of the Drinfel'd double was Abelian, the system of PDEs (12) and (13) separated and became solvable. Performability of the last step of the Poisson-Lie transformation, namely finding coordinates of the plural decompositions, depends critically on the complexity of the structure of Drinfel'd double where the $\sigma$-models live. In the investigated cases the Lie algebraic was simple enough so that the formula (29) for solution of (28) could be used and coordinates of the plural decompositions were found.

In the end we have investigated the conditions the must be applied to the $\sigma$-model solution in order that they satisfy the equations of motion for the relativistic strings. The conditions have very simple form for the flat models and they are preserved by the Poisson-Lie transformations. Therefore we were able to specify the type of $\sigma$-model solutions that are also solutions of equations of motion of three dimensional relativistic strings in the curved backgrounds. Examples were given in the section 5 .

\section{Acknowledgements}

This work was supported by the project of the Grant Agency of the Czech Republic No. 202/06/1480 and by the research plan LC527 15397/2005-31 of the Ministry of Education of the Czech Republic. The authors are grateful to Libor Šnobl for valuable comments. 


\section{References}

[1] L. Hlavatý, Classical solution of a sigma model in curved background, Phys. Lett. B 625 (2005) 285, hep-th/0506188.

[2] C. Klimčík and P. Ševera, Dual non-Abelian duality and the Drinfeld double, Phys. Lett. B 351 (1995) 455, hep-th/9502122.

[3] R. von Unge, Poisson-Lie T-plurality, J. High En. Phys. 02:07 (2002) 014, hep-th/00205245].

[4] L. Šnobl and L. Hlavatý, Classification of 6-dimensional real Drinfel'd doubles, Int.J.Mod.Phys. A17 (2002) 4043, math.QA/0202210.

[5] C. Klimčík, Poisson-Lie T-duality, Nucl. Phys. B (Proc. Suppl.) 46 (1996) 116, hep-th/9509095.

[6] L. Hlavatý, L. Šnobl, Poisson-Lie T-plurality of three-dimensional conformally invariant sigma models II : Nondiagonal metrics and dilaton puzzle, J. High En. Phys. 04:10(2004)045, hep-th/0408126.

[7] M. Turek, Geometric properties of dual $\sigma$-models, Diploma thesis, Prague 2005, hep-th/0512082. 